\title{
Competing or Interactive Effect Between Perceived Response Efficacy of Governmental Social Distancing Behaviors and Personal Freedom on Social Distancing Behaviors in the Chinese Adult General Population in Hong Kong
}

\author{
Yanqiu Yu ${ }^{\mathbb{(} \mathbb{D}}$, Joseph Tak Fai Lau ${ }^{q *(\mathbb{D})}$, Mason Man Chun Lau
}

\begin{abstract}
Background: Uptake of social distancing behaviors may be determined by a combination of individual perceptions and social values. The study investigated (1) the associations between individual perception of perceived response efficacy and social distancing behaviors, (2) the association between social value of perceived freedom infringement and social distancing behaviors, and (3) whether perceived freedom infringement would moderate the association between perceived response efficacy and social distancing behaviors.

Methods: A cross-sectional telephone survey interviewed 300 adults in the Hong Kong adult general population during April 21-28, 2020. The instruments of social distancing behaviors, perceived response efficacy, and perceived freedom infringement assessed the frequencies of practicing seven types of social distancing behaviors in the past week, perceived response efficacy of four types of governmental social distancing measures/instructions, and a 5-point Likert scale item on perceived infringement on personal freedom regarding a governmental social distancing measure of banning gatherings of $>4$ people in public areas. Linear regression adjusted for background factors was performed; the interaction term of perceived response efficacy $\times$ perceived freedom infringement was tested.

Results: About $40.4 \%-83.0 \%$ of the respondents practiced various types of social distancing behaviors; $57.3 \%-75.0 \%$ perceived response efficacies of related governmental measures; about $20 \%$ showed perceived freedom infringement. Perceived response efficacy, but not perceived freedom infringement, was independently and positively associated with social distancing behaviors. Perceived freedom infringement significantly moderated the association between perceived response efficacy and social distancing behaviors; such a positive association was significant at higher (those scored "extremely agree"), but not lower (those scored "extremely disagree"), levels of perceived freedom infringement. Conclusion: Perceived response efficacy is a potential determinant of social distancing. However, the strength of such an association may be modified by opposing social values about personal freedom. Future studies are warranted to verify above findings and explore other potential determinants.

Keywords: COVID-19, Social Distancing, Response Efficacy, Freedom, China, Interaction

Copyright: @ 2022 The Author(s); Published by Kerman University of Medical Sciences. This is an open-access article distributed under the terms of the Creative Commons Attribution License (https://creativecommons.org/licenses/ by/4.0), which permits unrestricted use, distribution, and reproduction in any medium, provided the original work is properly cited.

Citation: Yu Y, Lau JTF, Lau MMC. Competing or interactive effect between perceived response efficacy of governmental social distancing behaviors and personal freedom on social distancing behaviors in the Chinese adult general population in Hong Kong. Int J Health Policy Manag. 2022;11(4):498-507. doi:10.34172/ijhpm.2020.195
\end{abstract}

Article History:

Received: 26 September 2020 Accepted: 3 October 2020 ePublished: 18 October 2020

I Both authors contributed equally to this paper.

*Correspondence to: Joseph Tak Fai Lau Email: jlau@cuhk.edu.hk

\section{Background}

Social distancing has become one of the key global measures used to control the coronavirus disease 2019 (COVID-19) pandemic. ${ }^{1-6}$ The World Health Organization (WHO) suggests using the term "physical distancing" instead of "social distancing," as it is the physical distance that prevents transmission while people can remain socially connected via the Internet or other means. Social distancing had been used to reduce transmission of severe acute respiratory syndrome (SARS), swine flu (H1N1), and Ebola virus disease. ${ }^{7,8}$ In face of the high infectivity and asymptomatic transmission features of COVID-19, ${ }^{9,10}$ social distancing has particular significance. Social distancing during the COVID-19 pandemic includes multiple legal measures such as suspending classes/religious gatherings/sports events, banning people going out without important reasons, closing bars and restaurants, allowing people to work from home, and restricting size of gatherings (eg, four or eight) within distances of one to two meters. ${ }^{1-6}$ Together with the prevalent use of personal preventive measures (face-mask and good hand hygiene), social distancing should be able to curb the spread of the virus.

The effectiveness of the governmental social distancing measures, however, depends on the level of compliance in the general population, which cannot be taken for granted (eg, only around $40 \%$ in the United States according to a pre-print $\left.{ }^{11}\right)$. Keeping social distancing as part of a normal lifestyle is equally essential, as governmental social distancing measures cannot control all interpersonal contacts in public and private arenas. For instance, in many places, people are not required to stay at home (eg, many cities in China and the 


\section{Key Messages}

Implications for policy makers

- The levels of uptake of social distancing behaviors and perceived response efficacy of governmental social distancing measures were high in Hong Kong.

- Improvement on perceived efficacy of related governmental measures may increase the uptake of social distancing behaviors directly.

- Around one fifth of participants considered the restriction of gathering size in public areas an infringement of personal freedom.

- The effect of perceived infringement of freedom did not compete against perceived response efficacy onto the uptake of social distancing behaviors.

- The positive effect of perceived response efficacy on the uptake of social distancing behaviors was significant only among those with a stronger, but not weaker, perception about freedom infringement.

Implications for the public

Social distancing is a controversial public health measure that involves both public health benefits (eg, reduction of transmission of coronavirus disease 2019 [COVID-19]) and conflicting values (eg, perceived infringement of personal freedom). In Hong Kong SAR, China, the levels of perceived response efficacies were relatively high, which might contribute to the relatively high levels of the uptake of various social distancing behaviors. In contrast, about one fifth of participants had concerns about the infringement of personal freedom due to a social distancing measure, but such personal value had no competing effect against perceived response efficacy onto social distancing behaviors. Notably, perceived infringement of personal freedom moderated the association between perceived response efficacy and social distancing behaviors, indicating that perceived response efficacy only affected people who had stronger, but not weaker, perceptions about freedom infringement. The findings suggest that structural factors may modify the effect of cognitive factors on determining social distancing behaviors.

United States) and people are free to join large social gatherings in private settings. Thus, like other personal preventions, social distancing is very often a volitional personal choice that is subjected to multiple cognitive determinants. There is a dearth of published studies that investigated such determinants, although a few preprints reported factors such as income, empathy, norms, and boredom. ${ }^{12-15}$

Perceived response efficacy is a potential key factor of social distancing behaviors. It refers to a person's belief about whether the recommended action will actually avoid the threat. ${ }^{16}$ In the present study, it refers to the perceived effectiveness of the governmental social distancing policies in controlling the COVID-19 pandemic. Various behavioral health theories have highlighted the significance of perceived response efficacy in determining health-related behaviors. For instance, perceived response efficacy is often seen as a part of the construct of perceived benefits in the Health Belief Model, ${ }^{17}$ which states that a person would perform a health-related behavior if he/she perceives high susceptibility/ severity related to the health problem and high levels of perceived benefit/self-efficacy (confidence)/cue to action and a low level of barrier. The Protection Motivation Model of fear appeal also involved perceived response efficacy, which represents coping appraisal process regarding a threat; jointly with self-efficacy, it determines whether an individual would take up a health-related behavior. ${ }^{16}$ Empirically, ample studies have shown that perceived response efficacy of preventive measures was significantly associated with uptake of such behaviors during $\mathrm{H} 1 \mathrm{~N} 1$ and $\mathrm{H} 5 \mathrm{~N} 1$ outbreak periods. ${ }^{18-21}$ The very limited literature suggested that the same was probably true regarding social distancing during the COVID-19 pandemic. A Korean study showed that perceived response efficacy was positively associated with social distancing behaviors (reducing the use of public transportation, keeping away from crowded places, and postponing/canceling social events ${ }^{22}$; another Iranian study reported that perceived response efficacy was positively associated with protection motivation to conduct COVID-19-related preventive behaviors among health workers, which was associated with the actual behaviors. ${ }^{23}$ A study showed that perceived efficacy of governmental actions related to COVID-19 varied across countries (Norway > Israel >the United States >Colombia $>$ Brazil $>$ Germany), and it was associated with the number of individuals' preventive actions. ${ }^{24}$ Our literature search cannot find other studies that investigated the relationships between perceived response efficacy of governmental social distancing measures and individual social distancing behaviors related to COVID-19. Such studies are important as perceived response efficacy can be increased by health promotion.

According to the socio-ecological model, structural factors are also strong determinants of health-related behaviors. ${ }^{25-27}$ There were severe disputes about the implementation of social distancing measures within some governments and countries, such as the United States. ${ }^{6,28}$ This disputes were partly due to the obviously severe negative impacts of social distancing on the recessing economies in many countries. ${ }^{29}$ As a structural factor, inconsistency between social values and a public health control measure may reduce public engagement in the behavior (social distancing in this case). For instance, some studies conducted in Africa reported that trust toward the government was associated with preventive behaviors against Ebola. ${ }^{30-32} \mathrm{~A}$ few studies looked at the role of values and various community responses during the COVID-19 pandemic. One of them showed that collectivism was associated with a stronger risk perception of COVID-19 and weaker psychological maladjustment. ${ }^{33}$ Freedom is highly valued. A preprint reported that social distancing policies had reduced overall movement and travel at both individual and state levels in the United States. ${ }^{34}$ Social distancing, including the commonly exercised mandatory measure of gathering size restriction, may be perceived as an infringement of personal freedom in Hong Kong. It changed from a restriction of $>4$ people (from March 29 to May 4, 2020 when the study was conducted) to $>8$ people (from May 5 to June 18,2020 ) 
according to the severity of the outbreak, and it has a strong impact on Hong Kong people's daily life, as it is a very dynamic and densely populated city that people have to meet frequently for social and work reasons. The belief that social distancing infringes on individual freedom may compromise compliance with social distancing measures. In some countries (eg, the United States, Britain, and Italy), protests were held against the implementation of governmental social measures, stating that it limits personal freedom. ${ }^{35,36}$ In a previous study, the view that "the government interferes far too much in our everyday lives" was negatively associated with perceived risk for COVID-19. ${ }^{37}$ Our literature review, however, cannot identify any study that investigated the relationship between the value of personal freedom and social distancing behaviors.

The present study investigated the associations between a personal-level facilitating factor (perceived response efficacy of social distancing measures) and a structural prohibiting factor (the perception that the mandatory restriction of gathering size in public areas infringes personal freedom) and the levels of social distancing behaviors in the past week. Besides looking at whether the two constructs would exhibit independent competing associations with social distancing behaviors, statistical significance of the moderation effect of perceived infringement of personal freedom for the association between perceived response efficacy and levels of social distancing behaviors was also tested in this study. Three hypotheses were tested: (1) perceived response efficacy would be positively associated with social distancing behaviors; (2) perceived infringement of personal freedom would be negatively associated with social distancing behaviors; (3) a significant statistical interaction would exist (ie, the association between perceived response efficacy and social distancing behaviors would be stronger among those with higher levels than those with lower levels of perceived infringement of personal freedom).

\section{Methods \\ Study Design}

A random telephone survey was conducted among Hong Kong Chinese adults (aged $\geq 18$ years) during April 21-28, 2020; 300 participants were anonymously interviewed between 6-10:30 PM (10 to 15 minutes) by experienced interviewers to avoid over-sampling non-working individuals. Telephone numbers were randomly drawn from the most updated residential telephone directory. The household member whose birthday was closest to the interview date was invited to join the study if the household had more than one eligible prospective participants. The participants were briefed by the interviewer about the background of the study and the option to skip questions or quit any time without being questioned. The questions were asked in the fixed sequence of perceptions (perceived response efficacy and perceived infringement of personal freedom) followed by behaviors (social distancing). Unanswered telephone calls were given at least three attempts before being classified as invalid. Unavailable eligible participants were contacted again; appointments were made if necessary. Verbal informed consent was obtained prior to commencement of the interviewers; the interviewer signed on a form to plead that they had explained the study fully to the participants and answered their questions. Of the eligible participants, 54.3\% $(\mathrm{n}=300)$ completed the interviews. No incentives were given to the participants.

\section{Measures}

\section{Background Variables}

Information about socio-demographics and the perceived need to have close physical contacts during work ( $1=$ extremely low/not applicable to $5=$ extremely high) was collected.

\section{Social Distancing Behavior Scale}

The 7-item scale assessed the levels of social distancing behaviors in the past week, by asking about self-reported frequencies of avoiding: (i) going out unless necessary, (ii) social gatherings, (iii) meeting with acquaintances, (iv) visiting crowded places, $(v)$ being within 1.5 meters with other people, ( $v i$ ) gatherings involving $>4$ persons, and (vii) using public transportation. The items were measured with 5-point Likert scales ( $1=$ never to $5=$ always); higher scores indicated higher levels of social distancing behaviors. Confirmatory factor analysis was conducted to test the one-factor model of the Social Distancing Behavior Scale, which found satisfactory goodness-of-fit indices after taking into account of covariance between item error terms (Chi-square/ $d f=1.28, P<.001$, comparative fit index $[\mathrm{CFI}]=0.99$, normed fit index $[\mathrm{NFI}]=$ 0.98 , and root mean square error of approximation [RMSEA] $=0.07)$. The Cronbach's alpha was 0.82 .

\section{Perceived Response Efficacy Scale of Governmental Social Distancing Measures}

The 4-item scale assessed the level of perceived response efficacy for various governmental social distancing measures/ instructions, including $(i)$ banning gatherings of $>4$ persons in public areas, (ii) closure of entertainment venues (eg, bars, cinemas, beauty salons, night clubs, and fitness centers), (iii) closure of public facilities (eg, library and sports field), and (iv) banning people going out unless necessary. The items were rated with 5 -point Likert scales ( $1=$ extremely low to 5 = extremely high); higher scores indicated higher levels of perceived response efficacy. The confirmatory factor analysis on the one-factor model of the perceived response efficacy scale (PRES) showed satisfactory goodness-of-fit [Chi-square $/ d f=3.54, P<.001, \mathrm{CFI}=0.99, \mathrm{NFI}=0.99$, and RMSEA $=0.09]$. The Cronbach's alpha was 0.82 .

\section{Perceived Infringement of Personal Freedom Due to Social Distancing}

One item assessed how much the participants agreed with the statement "The banning of gatherings of $>4$ persons in public areas by the government infringes on my right for personal freedom" ( $1=$ extremely disagree to $5=$ extremely agree).

\section{Statistical Analysis}

Simple and multivariable (adjusted for background factors) ordinary least square linear regression analysis were conducted to test the associations among background 
factors, PRES, perceived infringement of freedom, and the level of social distancing behaviors. Standardized regression coefficients $(\beta)$ were presented in this report. It represents the change in the number of standard deviation (SD) in the dependent variable, given one unit change in the independent variable of concern. The moderation effects of perceived infringement of freedom on the association between PRES and social distancing behaviors were examined by testing the significance of the interaction term of PRES $\times$ perceived infringement of freedom, after adjustment of background factors. To illustrate the meaning of the moderation effect graphically, the modeled regression lines between perceived response efficacy and social distancing behaviors were plotted for two scores of the perceived freedom infringement scale with endorsement of "extremely disagree (value $=1$ )" and "extremely agree (value $=5$ )." The moderation effect and the graphical presentation were generated by Process $\mathrm{Macro}^{38}$ in SPSS 21.0. The statistically significant level was defined as two-sided $P<.050$. The term of marginal significance was used when the results were close to but did not meet the traditional convention for statistical significance $(P<.050)$, ie, when $.050<P<.100$.

\section{Results}

\section{Descriptive Statistics}

\section{Background Characteristics of the Participants}

Among the 300 participants, close to or more than half were females $(67.3 \%)$, aged $>55$ years old $(47.4 \%)$, and being married/cohabitating with someone (65.3\%). About $1 / 4$ received tertiary education or above $(25.6 \%)$ and perceived moderate to extremely high need to have close physical contacts $(<1.5$ meters) during work (26.7\%) (Table 1$)$.

\section{Social Distancing Behavior Scale}

The mean (SD; range) score of the Social Distancing Behavior Scale was 27.8 (5.7; 7-35). Descriptive statistics of compliance with specific social distancing behaviors are presented in Figure 1. Close to or more than $80 \%$ of the participants frequently or always avoided (i) going out unless necessary (78.0\%), (ii) social gatherings (78.7\%), (iii) meeting with acquaintances $(78.3 \%)$, (iv) visiting crowded places $(83.0 \%)$, $(v)$ being within $<1.5$ meters with other people $(67.4 \%)$, and ( $v i$ ) gatherings involving $>4$ persons (78.0\%). Fewer people, however, frequently/always avoided using public transportation $(40.4 \%)$.

\section{Perceived Response Efficacy of Governmental Social Distancing Measures}

The mean (SD; range) score of the PRES was 15.1 (3.5; 4-20). Over half of the participants considered specific governmental measures to be highly or extremely highly efficacious, including (i) banning gatherings of $>4$ persons (64.0\%), (ii) closure of entertainment venues (75.0\%), (iii) closure of public facilities $(65.7 \%)$, and (iv) banning people going out unless necessary (57.3\%) (Table 1).

\section{Perceived Infringement of Personal Freedom}

Nearly one-fifth of the participants agreed or extremely
Table 1. Descriptive statistics of participants $(n=300)$

\begin{tabular}{|c|c|c|}
\hline & No. & $\%$ \\
\hline \multicolumn{3}{|l|}{ Background Variables } \\
\hline \multicolumn{3}{|l|}{ Gender } \\
\hline Male & 98 & 32.7 \\
\hline Female & 202 & 67.3 \\
\hline \multicolumn{3}{|l|}{ Age } \\
\hline $18-35$ & 53 & 17.7 \\
\hline $36-55$ & 102 & 34.0 \\
\hline $56-65$ & 65 & 21.7 \\
\hline$>65$ & 77 & 25.7 \\
\hline Missing data & 3 & 1.0 \\
\hline \multicolumn{3}{|l|}{ Marital status } \\
\hline Single/separated/divorced/widow/widower & 104 & 34.7 \\
\hline Cohabitation/married & 196 & 65.3 \\
\hline \multicolumn{3}{|l|}{ Educational level } \\
\hline$\leq$ Primary school & 53 & 17.7 \\
\hline Middle school/matriculation & 169 & 56.3 \\
\hline$\geq$ College & 77 & 25.6 \\
\hline Missing data & 1 & 0.3 \\
\hline \multicolumn{3}{|l|}{$\begin{array}{l}\text { Perceived need to have close physical contacts } \\
\text { during work }\end{array}$} \\
\hline Not applicable/extremely low/low & 220 & 73.3 \\
\hline Moderate/high/extremely high & 80 & 26.7 \\
\hline \multicolumn{3}{|c|}{$\begin{array}{l}\text { Perceived Response Efficacy of Governmental Social Distancing } \\
\text { Measures }\end{array}$} \\
\hline \multicolumn{3}{|l|}{ Prohibition of gatherings of $>4$ persons } \\
\hline Extremely low & 9 & 3.0 \\
\hline Low & 19 & 6.3 \\
\hline Moderate & 80 & 26.7 \\
\hline High & 123 & 41.0 \\
\hline Extremely high & 69 & 23.0 \\
\hline \multicolumn{3}{|l|}{ Closure of entertainment venues } \\
\hline Extremely low & 7 & 2.3 \\
\hline Low & 14 & 4.7 \\
\hline Moderate & 54 & 18.0 \\
\hline High & 127 & 42.3 \\
\hline Extremely high & 98 & 32.7 \\
\hline \multicolumn{3}{|l|}{ Closure of public facilities } \\
\hline Extremely low & 11 & 3.7 \\
\hline Low & 34 & 11.3 \\
\hline Moderate & 58 & 19.3 \\
\hline High & 108 & 36.0 \\
\hline Extremely high & 89 & 29.7 \\
\hline \multicolumn{3}{|l|}{ Prohibition of people going out unless necessary } \\
\hline Extremely low & 29 & 9.7 \\
\hline Low & 32 & 10.7 \\
\hline Moderate & 67 & 22.3 \\
\hline High & 73 & 24.3 \\
\hline Extremely high & 99 & 33.0 \\
\hline \multicolumn{3}{|l|}{ Perceived Infringement of Personal Freedom } \\
\hline Extremely disagree & 78 & 26.0 \\
\hline Disagree & 107 & 35.7 \\
\hline Neutral & 58 & 19.3 \\
\hline Agree & 33 & 11.0 \\
\hline Extremely agree & 24 & 8.0 \\
\hline
\end{tabular}




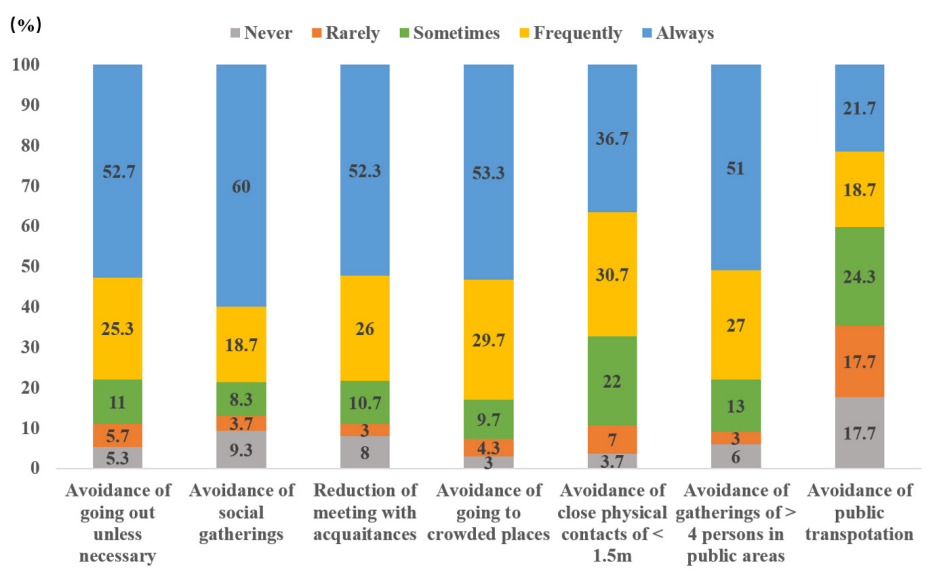

Figure 1. Distributions of the 7 Types of Social Distancing Behaviors.

agreed with the statement that banning gatherings involving $>4$ persons infringed on his/her right for personal freedom (19.0\%) (Table 1).

Simple Regression Analysis for the Associations Between Background Factors and Perceived Response Efficacy/ Perceived Infringement of Personal Freedom

The results are shown in Table 2. Age and current marital/ cohabitation status were positively associated with perceived response efficacy, while those with the secondary educational level were less likely than others to perceive response efficacy. Those with older age and perceived higher need to have close physical contacts during work were less likely than others to perceive infringement of personal freedom. The other associations were statistically non-significant. The background factors listed in Table 2 altogether explained $22.1 \%$ and $5.9 \%$ of the variance $\left(R^{2}\right)$ of perceived response efficacy and perceived infringement of personal freedom, respectively (statistics not shown in the tables).

Correlation Between the 2 Independent Variables

Perceived response efficacy was negatively correlated with perceived infringement of personal freedom $(r=-0.34$, $P<.001)$

Factors of Social Distancing Behaviors

The simple regression (one independent variable and one dependent variable in each model) results showed that two

Table 2. Simple Linear Regression Analysis Among the Studied Variables $(n=300)$

\begin{tabular}{|c|c|c|c|c|c|c|}
\hline & \multicolumn{2}{|c|}{$\begin{array}{c}\text { Perceived Response } \\
\text { Efficacy }\end{array}$} & \multicolumn{2}{|c|}{$\begin{array}{l}\text { Perceived Infringement of } \\
\text { Personal Freedom }\end{array}$} & \multicolumn{2}{|c|}{$\begin{array}{c}\text { Social Distancing } \\
\text { Behaviors }\end{array}$} \\
\hline & B & $P$ & 8 & $P$ & B & $P$ \\
\hline \multicolumn{7}{|l|}{ Background variables } \\
\hline \multicolumn{7}{|l|}{ Gender } \\
\hline Male & Ref & & Ref & & Ref & \\
\hline Female & -0.06 & .342 & -0.01 & .963 & 0.07 & .202 \\
\hline \multicolumn{7}{|l|}{ Age } \\
\hline $18-35$ & Ref & & Ref & & Ref & \\
\hline $36-55$ & 0.36 & $<.001$ & -0.14 & .075 & 0.11 & .135 \\
\hline $56-65$ & 0.34 & $<.001$ & -0.14 & .059 & 0.35 & $<.001$ \\
\hline$>65$ & 0.57 & $<.001$ & -0.23 & .003 & 0.21 & .004 \\
\hline \multicolumn{7}{|l|}{ Marital status } \\
\hline Single/separated/divorced/widow/widower & Ref & & Ref & & Ref & \\
\hline Cohabitation/married & 0.14 & .014 & -0.09 & .130 & 0.12 & .045 \\
\hline \multicolumn{7}{|l|}{ Educational level } \\
\hline$\leq$ Primary school & Ref & & Ref & & Ref & \\
\hline Middle school/matriculation & -0.25 & .001 & 0.03 & .693 & -0.10 & .203 \\
\hline$\geq$ College & -0.12 & .124 & 0.03 & .748 & -0.08 & .337 \\
\hline \multicolumn{7}{|c|}{ Perceived need to have close physical contacts during work } \\
\hline Not applicable/extremely low/low & Ref & & Ref & & Ref & \\
\hline Moderate/high/extremely high & 0.04 & .505 & -0.12 & .046 & -0.09 & .133 \\
\hline Perceived response efficacy & - & - & - & - & 0.19 & .001 \\
\hline Perceived infringement of personal freedom & - & - & - & - & -0.10 & .097 \\
\hline
\end{tabular}


of the background factors [older age ( $>55$ years versus 18-35 years) and current marital/cohabitation status] and perceived response efficacy were associated with higher levels of social distancing behaviors $(\beta=0.19 ; P=.001)$, while perceived infringement of personal freedom were inversely and marginally $(.050<P<.100)$ associated with social distancing behaviors $(\beta=-0.10 ; P=.097)$ (see Table 2$)$.

The results of the multivariable analysis that adjusted for all the background factors are presented in Table 3. First, perceived response efficacy was significantly and positively associated with social distancing behaviors $\left(R^{2}=0.115 ; \beta=\right.$ $0.15 ; P=.018$; Model 1). Second, the association between perceived infringement of personal freedom and social distancing behaviors was statistically non-significant $\left(R^{2}=\right.$ 0.103; $\beta=-0.07 ; P=.200$; Model 2). Third, when both perceived response efficacy and perceived infringement of personal freedom were present in the same model (Model 3), the adjusted analysis yielded a R-square value of 0.116 . In that model, perceived response efficacy $(\beta=0.14 ; P=.038)$ but not perceived infringement of personal freedom $(\beta=-0.63$; $P=.528$ ) was significantly associated with social distancing behaviors.

Testing Moderation Effect of Perceived Infringement of Personal Freedom on the Association Between Perceived Response Efficacy and Social Distancing Behaviors

In Model 4 of Table 3, the main effect of perceived infringement of personal freedom (the moderator) and the interaction term (perceived infringement $\times$ perceived response efficacy), but not the main effect of perceived response efficacy, were statistically significant. The graphical representation of the interaction effect is shown in Figure 2. The strength of the association between perceived response efficacy increased with the level of perceived infringement of personal freedom. Specifically, among those who "extremely agreed" with the statement about perceived infringement of freedom, perceived response efficacy was positively associated with social distancing behaviors $(B=0.621 ; P=.004)$; the association was non-significant among those who "extremely disagree" with the statement about perceived freedom infringement $(B$ $=0.006 ; P=.969)$. It is seen from Figure 2 that the slope of those with perceived infringement of personal freedom was substantial. It implies that an increase in 2.35 points in social distancing scale score (with the scale range 28 points of the
Social Distancing Behavior Scale) would be resulted when the PRES score increased by 3.5 points, which is equivalent to one $\mathrm{SD}$ (within the range of 16 points of PRES). In comparison, the slope of those who did not perceive freedom infringement, as seen from Figure 2, was almost flat.

\section{Discussion}

Social distancing has been used as a major global strategy to control the COVID-19 pandemic. The scale and scope of the related measures were unprecedented, ranging from lockdowns to restriction of gathering size in public areas, and involved fines and even imprisonment. The majority of the participants (about 3/4 or more) had practiced various means of social distancing (eg, avoiding social gatherings). However, less seemed to be able to practice physical distancing (eg, keeping a physical distance of $>1.5$ meters and avoiding using public transportation). This is understandable in the context of Hong Kong where there was no lockdown and working and daily activities were in general normal. The densely populated environment and reliance on public transportation limited the extent of physical distancing. Thus, the level of physical distancing in a city may depend on contextual factors such as population density and utilization rate of public transportation. It is important to compare the findings with other countries that vary in population density and other epidemic and policy features of COVID-19 (eg, the number of cases and the degree of lockdown). Policy-making on social distancing measures need to take the physical and social contexts into account.

In Hong Kong, the relatively low number of COVID-19 infection cases (1103 cases as of June 5, 2020) could be attributed to the almost universal facemask use, ${ }^{39,40}$ extensive screening, contact tracing, and quarantine policies. Voluntary and mandatory social distancing should have also played an important role as all these measures reinforce each other. Although social distancing was common, about $1 / 4$ or more of the participants did not observe such measures. Thus, health promotion is still necessary. Younger and single participants were less likely than their counterparts to practice social distancing as they may have more active social and work life. Sex and education were not significantly associated with social distancing. The reasons require further study, as such factors might be both positively and/or negatively associated with multiple factors of social distancing.

Table 3. Multivariable Linear Regression Analysis for Testing Factors of Social Distancing Behaviors and the Interaction Between the 2 Factors $(n=300)$

\begin{tabular}{|c|c|c|c|c|}
\hline & \multicolumn{4}{|c|}{ Social Distancing Behaviors } \\
\hline & Model 1 & Model 2 & Model 3 & Model 4 \\
\hline & $\beta$ & $\beta$ & $\beta$ & $\beta$ \\
\hline Perceived response efficacy & $0.15(P=.018)$ & & $0.14(P=.038)$ & $-0.09(P=.463)$ \\
\hline Perceived infringement of freedom & & $-0.07(P=.200)$ & $-0.63(P=.528)$ & $-0.51(P=.026)$ \\
\hline Perceived response efficacy $\times$ Perceived infringement of freedom & & & & $0.46(P=.032)$ \\
\hline$d f 1, d f 2$ & $(9,290)$ & $(9,290)$ & $(10,289)$ & $(11,288)$ \\
\hline$F$ & $4.196(P<.001)$ & $3.706(P<.001)$ & $3.809(P<.001)$ & $3.927(P<.001)$ \\
\hline$R^{2}$ & 0.115 & 0.103 & 0.116 & 0.130 \\
\hline
\end{tabular}




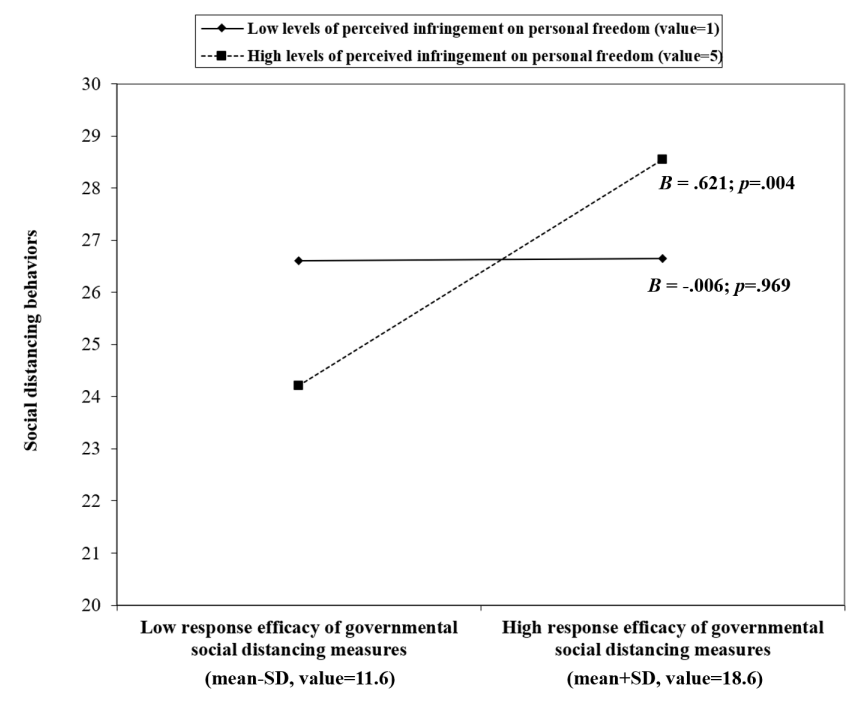

Figure 2. Graphical Representation of the Moderation Effect. Abbreviation: $\mathrm{SD}$, standard deviation.

More than half of the participants perceived relatively high response efficacy regarding various public health measures. About $3 / 5$ to $3 / 4$ of the participants endorsed response efficacy of restricting gathering size of $\leq 4$, closure of entertainment venues and public facilities (eg, playgrounds and museums), and avoidance of going out unless necessary. It is imperative that good compliance of such policies requires good perceived response efficacy in the general population. In the present study, it is noteworthy that perceived response efficacy of the social distancing measures was significantly associated with the level of social distancing. The findings corroborated previous studies that response efficacy of public health measures may increase levels of uptake of personal preventive behaviors. ${ }^{18-21}$ The study adds similar evidence to the literature of COVID-19 studies. It also partially supports related behavioral health theories postulating perceived response efficacy as a determinant of preventive behaviors (eg, the Health Belief model). International comparisons are important as the levels of perceived response efficacy seem to vary tremendously across countries and among political leaders, who faced tough decisions of balancing between social distancing and economic recovery.

Given the positive association between perceived response efficacy and social distancing behaviors, future studies should explore whether fostering perceived response efficacy via health promotion would increase social distancing during the COVID-19 pandemic. If the findings were affirmative, improvement could be made as the endorsement rate of perceived response efficacy was only about $2 / 5$ in this study. Such health promotion may need to pay attention to the younger people and those with secondary education level as they tended to report lower perceived response efficacy. Younger people might perceive low response efficacy due to their lower perceived risk and perceived susceptibility regarding COVID-19.41 Besides, younger people in Hong Kong showed very low levels of trust toward the government due to the ongoing social movement (unpublished data). The lack of trust might have weakened young people's perceived response efficacy.

Furthermore, we need to know more about the actual response efficacy of social distancing measures, as, except for a few observational studies conducted during $\mathrm{H} 1 \mathrm{~N} 1$ and H5N1 outbreaks, ${ }^{18-21}$ solid evidence about the efficacy of social distancing in controlling outbreaks of emerging infectious diseases seems unavailable. It is noteworthy that the setting up and/or relaxation of social distancing rules (eg, one-totwo-meter physical distancing and gathering size of $\leq 4 / \leq 8$ ) might actually be arbitrary and certainly not evidence-based. Although some related modeling data looked at the effect of social distancing on the spread of COVID-19, ${ }^{11,42}$ contextual factors (eg, compliance, screening policies, and the number of cases) might affect the models' accuracies. It is also warranted to understand the effectiveness of various means of social distancing in populations that have various facemask usage rates.

Health promotors need to take structural factors that facilitate or compromise social distancing behaviors into consideration. It is noteworthy that about $1 / 5$ of the participants believed that the local policy of banning gatherings of size $>4$ in public areas had infringed their freedom. Understandably, perceived freedom infringement was found to be negatively associated with perceived response efficacy. First, according to cognitive emotion regulation theory, those who perceived infringement of freedom may have negative attitudes about social distancing; the negative emotion may foster negative cognitions about the social distancing measures. ${ }^{43}$ Second, age may be a potential confounder as younger people tended to perceive both lower response efficacy and stronger infringement of personal freedom. Third, the concern about personal freedom might be even stronger in Western societies, which has a tradition of emphasizing the protection of personal freedom. ${ }^{44}$ It is interesting to point out that Hong Kong has cultural heritages from both the East and the West. A majority (90.6\%) of the Hong Kong residents are Chinese; most of their parents or grandparents immigrated from mainland China during the 1950s and 1960s. Traditional Chinese culture is thus vital. In parallel, the city was a British colony prior to 1997. As a result, the education, legal, and medical systems were (are) under strong British influences. Hong Kong has also evolved herself into one of the most internationalized cities. Thus, both the traditional Chinese collectivism and the western belief of personal freedom have deep roots in the general population. It is possibly that Hong Kong has a 'middle ground' position in the spectrum of individualism versus collectivism. Overall, we thus believe that perception of freedom infringement related to social distancing and its impact on social distancing in the Hong Kong general population might be stronger than those of some traditional Asian countries but weaker than those of the Western countries. Cross-cultural comparisons are potentially useful.

It is interesting to see that perceived infringement of personal freedom was not directly associated with social distancing behaviors. It thus did not point in the opposite direction against perceived response efficacy in determining levels of social distancing. One plausible reason for the non- 
significant association between perceived infringement of freedom and social distancing was that, as previously mentioned, Hong Kong Chinese people are strongly influenced by the traditional Chinese culture that emphasizes collectivism. Some aspects of collectivism have been reported to be associated with specific health-related behaviors (eg, risky sexual behaviors) in previous studies. ${ }^{45,46}$ It may partially explain the cross-country variations in the uptake of preventive measures against COVID-19 (such as social distancing) and the degree of perceived freedom infringement regarding social distancing. Some people might practice social distancing even if they feel that the measure has some infringements of their freedom because of collectivism. Some degrees of personal sacrifice for social good is the norm among Chinese people. ${ }^{47}$ Collectivism seems to have been well observed in China during the pandemic; an illustration is the great support shown to the government despite personal inconvenience during the lockdowns. ${ }^{48}$

Despite the non-significant association with social distancing behaviors, interestingly, perceived infringement of freedom moderated the significant association between perceived response efficacy and social distancing behaviors. The moderation effect elaborates the relationship between perceived response efficacy and social distancing behaviors. The association was significant only among those who perceived infringement of freedom, but not among those who did not. The data suggest that perceived response efficacy might not influence all people. It might only matter in the presence of a value conflict regarding social distancing. It is plausible that those who possessed a negative value (infringement on personal freedom in this case) might need a stronger reason (perceived response efficacy) to initiate social distancing behaviors. It is noteworthy that the relationship between perceived response efficacy and social distancing might actually depend on the presence of a potentially inherent value conflict (a structural factor) regarding social distancing. Future studies may look at whether other structural moderators (eg, financial loss related to social distancing and collectivism) exist for the association between perceived response efficacy (and also other cognitive factors) and social distancing. However, readers are reminded that the moderation analysis was preliminary and exploratory in nature, and needs to be confirmed by future studies.

The study has some limitations. First, the study has some methodological limitations. (1) We cannot make causal inferences as this was a cross-sectional study. (2) Social desirability bias and recall bias may exist. (3) The observed effect size of the associations between perceived response efficacy/perceived infringement of personal freedom and social distancing was small ( $R^{2}$ ranged from $\left.10 \%-13 \%\right)$. The finding implies that some important factors might not have been included in the present study (eg, perceived risk and perceived susceptibility related to COVID-19, and variables related to social distancing, such as self-efficacy, boredom, and social norms). (4) The response rate was not high, although being comparable to other telephone surveys conducted in Hong Kong ${ }^{49,50}$; characteristics between participants and nonparticipants may differ. (5) The sample's age distribution and education levels were similar to those of the census, ${ }^{51}$ although there were slight differences. For instance, the 18-35 age group was under-represented (17.7\% versus $22.8 \%$ ), while the $>65$ age group was slightly over-represented (25.7\% versus $21.2 \%)$ in the sample. Besides, people with tertiary education were slightly under-represented in our sample when compared to the census data (25.6\% versus $33.1 \%)$. Slight biases may exist, which should be normal. (6) The sequence of the question items was fixed and there might be an order effect. Second, some limitations refer to variable selection: (1) The study did not investigate the employment status of participants, which may be an important socio-demographical factor of social distancing. (2) It did not investigate other potential moderators, such as self-control, collectivism, and financial loss due to COVID-19. Third, some contextual limitations are also noteworthy: (1) Perceived infringement of personal freedom was measured by a single item about whether the restriction on gathering size of $>4$ persons in public areas would infringe personal freedom as the other commonly adopted mandatory governmental social distancing behaviors (eg, stay-at-home order, working from home, and closure of shops and restaurants) were not in place in Hong Kong during the study period. (2) Hong Kong is undergoing sociopolitical movements and turmoil prior to and during the COVID-19 pandemic, the social distancing measures might have been conflated with the heated political sentiments that the measures were intended to limit political freedom (eg, the right to protest). Interpretations need to take such processes into account.

\section{Conclusion}

We found high levels of various types of social distancing behaviors and relatively high levels of perceived response efficacy. About $1 / 5$ of the participants were concerned about the infringement of personal freedom due to a social distancing measure. It is interesting that perceived response efficacy did not compete (opposite effects) against each other to affect social distancing. Instead, a significant moderation effect was found. In short, social distancing may involve both public health benefits (eg, perceived response efficacy) and conflicting values (eg, perceived infringement of freedom). It is important to elaborate on the roles of the conflicting values in the relationship between cognitive factors and social distancing behaviors. There is a dearth of similar studies that investigated the role of structural factors and social values in determining social distancing, or how such factors would modify the effects of cognitive factors on social distancing. Such studies are important to understand and promote social distancing to control COVID-19 as it is a controversial public health measure. Social distancing is a new and understudied area of public health research that requires applications of interdisciplinary research frameworks. Cross-cultural studies are of particular importance as values related to COVID-19 (eg, freedom) may be tremendously different across countries; these studies may involve other theoretical frameworks (eg, the Social Cognitive Theory and Self-determination Theory). Given individual variations in collectivism, future studies should also investigate its relationship with perceived 
freedom infringement, and whether collectivism would serve as another potential moderator of the association between perceived response efficacy and social distancing behaviors. It is a limitation that it was not assessed in the present study.

All in all, it is important to understand social distancing and related perceptions in their socio-political contexts. The pandemic and its related measures, both governmental and personal ones, have been strongly politicized in the United States and other countries, ${ }^{52-54}$ where polarization toward the use of such measures was severe and social distancing and face-mask use have been used as symptoms of political stance. In Hong Kong, various opposing political parties have been criticizing many governmental measures for controlling COVID-19, such as receiving support for largescale testing from the mainland Chinese government. Some politicians criticized the gathering size restriction due to economic reasons, but political parties were basically not divided in the views regarding the gathering size restriction policy. The political impacts on the compliance with social distancing measures in Hong Kong might be lower than that of the United States. However, due to the ongoing series of socio-political unrest, ${ }^{55,56}$ the public's trust in the government has dived deeply. It is possible that the lack of trust and politicization might reduce perceived response efficacy and its effect on mandatory social distancing. Future studies about social distancing in the COVID-19 context need to take the political context into account. Politicization of the pandemic and community response are hence a potentially important and new research direction.

\section{Acknowledgement}

We would like to thank all the participants for their contributions.

\section{Ethical issues}

The study was approved by the Survey and Behavioral Research Ethics Committee of the Chinese University of Hong Kong (No. SBRE-19-661)

\section{Competing interests}

Authors declare that they have no competing interests.

\section{Authors' contributions}

Conceptualization: JTFL and YQY; Methodology: YQY and JTFL; Investigation: MMCL; Software: YQY; Formal analysis: YQY; Data curation: YQY; Validation: JTFL; Resources: JTFL; Writing-original draft: YQY and JTFL; Writing-review \& editing: YQY and JTFL; Supervision: JTFL; Funding acquisition: JTFL.

\section{Funding}

The study was supported by internal research funding of the Centre for Health Behaviour Research. The funding source has no role in this study.

\section{References}

1. Wilder-Smith A, Freedman DO. Isolation, quarantine, social distancing and community containment: pivotal role for old-style public health measures in the novel coronavirus (2019-nCoV) outbreak. J Travel Med. 2020;27(2). doi:10.1093/jtm/taaa020

2. Lewnard JA, Lo NC. Scientific and ethical basis for social-distancing interventions against COVID-19. Lancet Infect Dis. 2020;20(6):631633. doi:10.1016/s1473-3099(20)30190-0

3. Flaxman S, Mishra S, Gandy A, et al. Estimating the effects of nonpharmaceutical interventions on COVID-19 in Europe. Nature. 2020; 584(7820):257-261. doi:10.1038/s41586-020-2405-7

4. Lau H, Khosrawipour V, Kocbach $P$, et al. The positive impact of lockdown in Wuhan on containing the COVID-19 outbreak in China. $J$
Travel Med. 2020;27(3). doi:10.1093/jtm/taaa037

5. Leung $\mathrm{K}$, Wu JT, Liu D, Leung GM. First-wave COVID-19 transmissibility and severity in China outside Hubei after control measures, and second-wave scenario planning: a modelling impact assessment. Lancet. 2020;395(10233):1382-1393. doi:10.1016/ s0140-6736(20)30746-7

6. Stawicki SP, Jeanmonod R, Miller AC, et al. The 2019-2020 Novel Coronavirus (Severe Acute Respiratory Syndrome Coronavirus 2) Pandemic: A Joint American College of Academic International Medicine-World Academic Council of Emergency Medicine Multidisciplinary COVID-19 Working Group Consensus Paper. J Glob Infect Dis. 2020;12(2):47-93. doi:10.4103/jgid.jgid_86_20

7. Fong MW, Gao $\mathrm{H}$, Wong JY, et al. Nonpharmaceutical measures for pandemic influenza in nonhealthcare settings-social distancing measures. Emerg Infect Dis. 2020;26(5):976-984. doi:10.3201/ eid2605.190995

8. Rothstein MA. From SARS to Ebola: legal and ethical considerations for modern quarantine. Indiana Health Law Rev. 2015;12(1):227-280. doi:10.2139/ssrn.2499701

9. $\mathrm{Hu}$ Z, Song C, Xu C, et al. Clinical characteristics of 24 asymptomatic infections with COVID-19 screened among close contacts in Nanjing, China. Sci China Life Sci. 2020;63(5):706-711. doi:10.1007/s11427020-1661-4

10. Al-Tawfiq JA. Asymptomatic coronavirus infection: MERS-CoV and SARS-CoV-2 (COVID-19). Travel Med Infect Dis. 2020;35:101608. doi:10.1016/j.tmaid.2020.101608

11. Moore RC, Lee A, Hancock JT, Halley M, Linos E. Experience with social distancing early in the COVID-19 pandemic in the United States: implications for public health messaging. medRxiv. 2020. doi: 10.1101/2020.04.08.20057067

12. Farias JEM, Pilati R. Violating social distancing amid COVID-19 pandemic: psychological factors to improve compliance. PsyArXiv. 2020. doi:10.31234/osf.io/apg9e

13. Kavanagh NM, Goel RR, Venkataramani AS. Association of countylevel socioeconomic and political characteristics with engagement in social distancing for COVID-19. medRxiv. 2020. doi:10.1101/2020.04 .06 .20055632

14. Pfattheicher S, Nockur L, Böhm R, Sassenrath C, Petersen MB. The emotional path to action: empathy promotes physical distancing and wearing of face masks during the COVID-19 pandemic. Psychol Sci. 2020. doi:10.1177/0956797620964422

15. Wolff W, Martarelli CS, Schüler J, Bieleke M. High boredom proneness and low trait self-control impair adherence to social distancing guidelines during the COVID-19 pandemic. Int J Environ Res Public Health. 2020;17(15). doi:10.3390/ijerph17155420

16. Rogers RW. A protection motivation theory of fear appeals and attitude change. J Psychol. 1975;91(1):93-114. doi:10.1080/002239 80.1975.9915803

17. Janz NK, Becker MH. The health belief model: a decade later. Health Educ Q. 1984;11(1):1-47. doi:10.1177/109019818401100101

18. Lau JT, Kim JH, Tsui H, Griffiths S. Perceptions related to human avian influenza and their associations with anticipated psychological and behavioral responses at the onset of outbreak in the Hong Kong Chinese general population. Am J Infect Control. 2007;35(1):38-49. doi:10.1016/j.ajic.2006.07.010

19. Lau JT, Griffiths S, Choi KC, Lin C. Prevalence of preventive behaviors and associated factors during early phase of the H1N1 influenza epidemic. Am J Infect Control. 2010;38(5):374-380. doi:10.1016/j. ajic.2010.03.002

20. de Zwart O, Veldhuijzen IK, Richardus JH, Brug J. Monitoring of risk perceptions and correlates of precautionary behaviour related to human avian influenza during 2006-2007 in the Netherlands: results of seven consecutive surveys. BMC Infect Dis. 2010;10:114. doi:10.1186/1471-2334-10-114

21. Bults M, Beaujean DJ, de Zwart O, et al. Perceived risk, anxiety, and behavioural responses of the general public during the early phase of the Influenza A (H1N1) pandemic in the Netherlands: results of three consecutive online surveys. BMC Public Health. 2011;11:2. doi:10.1186/1471-2458-11-2

22. Lee M, You M. Psychological and behavioral responses in South Korea during the early stages of coronavirus disease 2019 (COVID-19). Int J Environ Res Public Health. 2020;17(9). doi:10.3390/ijerph17092977 
23. Bashirian S, Jenabi E, Khazaei S, et al. Factors associated with preventive behaviours of COVID-19 among hospital staff in Iran in 2020: an application of the Protection Motivation Theory. J Hosp Infect. 2020;105(3):430-433. doi:10.1016/j.jhin.2020.04.035

24. Mækelæ MJ, Reggev N, Dutra N, et al. Perceived efficacy of COVID-19 restrictions, reactions and their impact on mental health during the early phase of the outbreak in six countries. $R$ Soc Open Sci. 2020;7(8):200644. doi:10.1098/rsos.200644

25. Bronfenbrenner U. Toward an experimental ecology of human development. Am Psychol. 1977;32(7):513-531. doi:10.1037/0003066x.32.7.513

26. Bronfenbrenner U. Ecology of the family as a context for human development: research perspectives. Dev Psychol. 1986;22(6):723742. doi:10.1037/0012-1649.22.6.723

27. Bronfenbrenner U. Ecological systems theory. In: Vasta R, ed. Six Theories of Child Development: Revised Formulations and Current Issues. Jessica Kingsley Publishers; 1992.

28. Briscese G, Lacetera N, Macis M, Tonin M. Compliance with COVID-19 Social-Distancing Measures in Italy: The Role of Expectations and Duration. Cambridge: National Bureau of Economic Research; 2020.

29. CNN News. A 'Short, Sharp' Global Recession is Starting to Look Inevitable. CNN News. March 10, 2020. https://edition.cnn. com/2020/03/09/economy/global-recession-coronavirus/index.html. Accessed May 7, 2020.

30. Blair RA, Morse BS, Tsai LL. Public health and public trust: survey evidence from the Ebola Virus Disease epidemic in Liberia. Soc Sci Med. 2017;172:89-97. doi:10.1016/j.socscimed.2016.11.016

31. Morse B, Grépin KA, Blair RA, Tsai L. Patterns of demand for nonEbola health services during and after the Ebola outbreak: panel survey evidence from Monrovia, Liberia. BMJ Glob Health. 2016; 1(1):e000007. doi:10.1136/bmjgh-2015-000007

32. Vinck P, Pham PN, Bindu KK, Bedford J, Nilles EJ. Institutional trust and misinformation in the response to the 2018-19 Ebola outbreak in North Kivu, DR Congo: a population-based survey. Lancet Infect Dis. 2019;19(5):529-536. doi:10.1016/s1473-3099(19)30063-5

33. Germani A, Buratta L, Delvecchio E, Mazzeschi C. Emerging adults and COVID-19: the role of individualism-collectivism on perceived risks and psychological maladjustment. Int J Environ Res Public Health. 2020;17(10). doi:10.3390/ijerph17103497

34. Gollwitzer A, Martel C, Marshall J, Höhs JM, Bargh JA. Connecting self-reported social distancing to real-world behavior at the individual and US state level. PsyArXiv. 2020. doi:10.31234/osf.io/kvnwp

35. Protests Over Responses to the COVID-19 Pandemic. Wikipedia website. https://en.wikipedia.org/wiki/Protests_over_responses_to the COVID-19 pandemic. Accessed June 4, 2020.

36. Dyer O. Covid-19: Trump stokes protests against social distancing measures. BMJ. 2020;369:m1596. doi:10.1136/bmj.m1596

37. Dryhurst S, Schneider CR, Kerr J, et al. Risk perceptions of COVID-19 around the world. J Risk Res. 2020:1-13. doi:10.1080/13669877.202 0.1758193

38. Hayes AF. Introduction to Mediation, Moderation, and Conditional Process Analysis: A Regression-Based Approach. New York, NY: The Guilford Press; 2018.

39. Cheng VC, Wong SC, Chuang VW, et al. The role of communitywide wearing of face mask for control of coronavirus disease 2019 (COVID-19) epidemic due to SARS-CoV-2. J Infect. 2020;81(1):107-
114. doi:10.1016/j.jinf.2020.04.024

40. Kwok KO, Li KK, Chan $\mathrm{HHH}$, et al. Community responses during early phase of COVID-19 epidemic, Hong Kong. Emerg Infect Dis. 2020; 26(7):1575-1579. doi:10.3201/eid2607.200500

41. He S, Chen S, Kong L, Liu W. Analysis of risk perceptions and related factors concerning COVID-19 epidemic in Chongqing, China. J Community Health. 2020:1-8. doi:10.1007/s10900-020-00870-4

42. Wellenius GA, Vispute S, Espinosa V, et al. Impacts of state-level policies on social distancing in the united states using aggregated mobility data during the covid-19 pandemic. arXiv. 2020.

43. Gross JJ. Emotion regulation: current status and future prospects. Psychol Inq. 2015;26(1):1-26. doi:10.1080/1047840x.2014.940781

44. Blokland HT. Freedom and Culture in Western Society. Vol 5. Psychology Press; 1997.

45. Pokhrel $\mathrm{P}$, Bennett BL, Regmi S, et al. Individualism-collectivism, social self-control and adolescent substance use and risky sexual behavior. Subst Use Misuse. 2018;53(7):1057-1067. doi:10.1080/10 826084.2017.1392983

46. Travaglino GA, Moon C. Explaining compliance with social distancing norms during the COVID-19 pandemic: the roles of cultural orientations, trust and self-conscious emotions in the US, Italy, and South Korea. PsyArXiv. 2020. doi:10.31234/osf.io/8yn5b

47. Wong YT. The Chinese at work: collectivism or individualism? Asian Profile. 2001;30(4):279-287.

48. Wu C. How Chinese Citizens View Their Government's Coronavirus Response. The Conversation. May 27, 2020. https://theconversation. com/how-chinese-citizens-view-their-governments-coronavirusresponse-139176. Accessed August 25, 2020.

49. Lau JT, Yang $\mathrm{X}$, Tsui $\mathrm{H}$, Kim JH. Monitoring community responses to the SARS epidemic in Hong Kong: from day 10 to day 62. J Epidemiol Community Health. 2003;57(11):864-870. doi:10.1136/jech.57.11.864

50. Lau JT, Tsui HY, Wang QS. Effects of two telephone survey methods on the level of reported risk behaviours. Sex Transm Infect. 2003; 79(4):325-331. doi:10.1136/sti.79.4.325

51. Census and Statistics Department. Women and Men in Hong Kong: Key Statistics. Hong Kong: Census and Statistics Department; 2019.

52. Rothgerber H, Wilson TL, Whaley D, et al. Politicizing the COVID-19 pandemic: ideological differences in adherence to social distancing. PsyArXiv. 2020. doi:10.31234/osf.io/k23cv

53. Abbas $\mathrm{AH}$. Politicizing the pandemic: a schemata analysis of COVID-19 news in two selected newspapers. Int J Semiot Law. 2020. doi:10.1007/s11196-020-09745-2

54. Ward J, Alleaume C, Peretti-Watel P. The French public's attitudes to a future COVID-19 vaccine: the politicization of a public health issue. SocArXiv. 2020. doi:10.31235/osf.io/xphe9

55. Lau JTF, Kim Y, Wu AMS, Wang Z, Huang B, Mo PKH. The Occupy Central (Umbrella) movement and mental health distress in the Hong Kong general public: political movements and concerns as potential structural risk factors of population mental health. Soc Psychiatry Psychiatr Epidemiol. 2017;52(5):525-536. doi:10.1007/s00127-0171340-X

56. Ni MY, Yao XI, Leung KSM, et al. Depression and post-traumatic stress during major social unrest in Hong Kong: a 10-year prospective cohort study. Lancet. 2020;395(10220):273-284. doi:10.1016/s01406736(19)33160-5 